\title{
Diseño y validación del perfil profesional de la Educación Especial: desarrollo metodológico
}

\author{
Design and validation of the professional profile of Special Education: \\ methodological development
}

Julieta Solórzano

julieta.solorzano@ucr.ac.cr

Universidad de Costa Rica, Costa Rica

Marcelo Rodríguez

mrodriguezag@ucatolica.ac.cr

Universidad Católica de Costa Rica, Costa Rica

\author{
Viviana Abarca \\ viviana.abarca@cenarec.go.cr \\ Centro Nacional de Recursos para la Educación \\ Inclusiva, Costa Rica
}

\section{Rocío Deliyore}

maria.deliyorevega@ucr.ac.cr

Universidad de Costa Rica, Costa Rica

Evelyn Hernández

ehernandezs@uned.ac.cr

Universidad Estatal a Distancia, Costa Rica

\section{Lady Meléndez}

lady_melendez@yahoo.com.mx

Universidad de Costa Rica, Costa Rica

Jorge Quesada

jquesada@colypro.com

Colegio de Licenciados y Profesores en Letras,

Filosofía, Ciencias y Artes, Costa Rica

\section{Rocío Ramírez}

rramirez@colypro.com

Colegio de Licenciados y Profesores en Letras,

Filosofía, Ciencias y Artes, Costa Rica

\author{
Mary Rojas \\ mary_rp_@hotmail.com \\ Sistema Nacional de Acreditación de la Educación \\ Superior, Costa Rica
}

Resumen: En una sociedad en constante cambio las demandas laborales cada vez exigen más habilidades y competencias por parte de los profesionales egresados de los programas de educación superior. La Educación Especial (EE) no escapa a esta realidad, por el contrario, las contribuciones de los Derechos Humanos, la Educación para Todos y la Educación Inclusiva obligan a repensar el rol de estos profesionales desde la epistemología misma de la disciplina. Es así como el equipo de investigadores del Observatorio Nacional para la Educación Inclusiva de Costa Rica (ONEI), se propuso establecer un perfil guía que sirviera como base para el planteamiento de planes de estudio, manuales de puestos, sistemas de contratación y evaluación, entre otros usos posibles. Este proceso tuvo una duración de dos años, implicó la participación de 14 investigadores y más de 800 personas informantes y validadoras a lo largo de las tres fases y cinco etapas de trabajo e intercambio entre metodologías cualitativas y cuantitativas; lo que permitió la construcción, reconstrucción y validación de un robusto perfil profesional para la Educación Especial costarricense. Este artículo expone precisamente la metodología desarrollada para el proceso de diseño y validación del perfil, con el propósito de que sirva de modelo para futuras réplicas en otras disciplinas, lo que también favorecerá la continuidad de la investigación a fin de validar su contenido con otras poblaciones, como las personas usuarias de los servicios de EE u otros profesionales afines, con quienes tradicionalmente se comparten los procesos educativos en los entornos inclusivos.

Palabras clave: Formación de docentes de educación especial, Educación especial, Educación inclusiva, Rol del profesor.

Abstract: In a constantly changing society, job demands increasingly demand more skills and competencies from professionals graduated from higher education programs. Special Education does not escape this reality, on the contrary, the contributions of Human Rights, Education for All and Inclusive Education force us to rethink the role of these professionals from the very epistemology of the discipline. Thus, the team of researchers from the National Observatory for Inclusive 


\author{
Mario Segura \\ mario.segura.castillo@mep.go.cr \\ Ministerio de Educación Pública, Costa Rica \\ Zarelly Sibaja \\ zsibaja@uned.ac.cr \\ Universidad Estatal a Distancia, Costa Rica
}

Gabriela Solís

gsolis.una@gmail.com

Universidad Nacional, Costa Rica

Marie Clare Vargas

marie_d_claire@yahoo.com

Universidad Nacional, Costa Rica

Yesenia Williams

ywilliams@ucatolica.ac.cr

Universidad Católica de Costa Rica, Costa Rica

Sandra Zúñiga

szuniga@sinaes.ac.cr

Sistema Nacional de Acreditación de la Educación

Superior, Costa Rica

Recepción: 09 Marzo 2021

Aprobación: 27 Mayo 2021

Publicación: 01 Junio 2021
Education of Costa Rica (ONEI) proposed to establish a guiding profile that would serve as a basis for the approach of study plans, job manuals, hiring and evaluation systems, among other possible uses. This process lasted two years, involved the participation of 14 researchers and more than 800 validating and informants throughout the three phases and five stages of work and exchange between qualitative and quantitative methodologies; which allowed the construction, reconstruction and validation of a robust professional profile for Costa Rican Special Education.

This article exposes precisely the methodology developed for the profile design and validation process, with the purpose of serving as a model for future replications in other disciplines, which will also favor the continuity of the research in order to validate its content with other populations, such as users of EE services or other related professionals, with whom educational processes are traditionally shared in inclusive settings.

Keywords: Special education teacher training, Special education, Inclusive education, Teacher role.

Cita sugerida: Solórzano, J. Rodríguez, M., Abarca, V., Deliyore, R., Hernández, E., Meléndez, L., Quesada, J., Ramírez, R., Rojas, M., Segura, M., Sibaja, Z., Solís, G., Vargas, M. C., Williams, Y. y Zúñiga, S. (2021). Diseño y validación del perfil profesional de la Especial: desarrollo metodológico. Revista Latinoamericana de Metodología de las Ciencias Sociales, 11(1), e090. https://doi.org/10.24215/18537863e090

\section{INTRODUCCIÓN}

El Observatorio Nacional de la Educación Inclusiva en Costa Rica (ONEI en adelante), integrado por personas representantes de diversas instituciones vinculadas con la gestión y la calidad educativa en el país, la formación de profesionales en el campo de la Educación Especial y la defensa del ejercicio profesional, se dio a la tarea de conceptualizar el perfil profesional del profesorado de Educación Especial (EE en adelante).

El país ha realizado acciones decididas por el desarrollo de una educación inclusiva y, en este contexto, el equipo reflexionó sobre el rol que tendría que asumir el profesorado de EE, por lo tanto, el diseño del perfil profesional debía considerar las demandas de la educación inclusiva, equitativa y de calidad, a la vez que permitiera derivar instrumentos para el reclutamiento y la selección profesional, el establecimiento de estándares mínimos e indicadores para la evaluación del desempeño docente y la toma de decisiones en atención al mejoramiento. También debía ser un marco orientador para el planteamiento, autoevaluación y acreditación de planes de formación y de programas de desarrollo profesional en el área.

Entre 2018 y 2020, mediante una aproximación mixta se construyó el perfil profesional, este documento comparte la metodología realizada y se caracteriza el perfil como posible insumo para otros ejercicios 
similares. En otra publicación se profundiza el contenido conceptual de cada una de las categorías que componen el perfil (Meléndez et al. 2020).

Para este proceso de definición del perfil profesional fue necesario realizar una revisión documental de lo existente en otros países, referida a los planes de estudio de las carreras de EE, las consultas a expertos y el análisis de trabajos de investigación referidos a la temática.

En relación con el estado del arte sobre las nuevas demandas a la formación del profesional de la EE, identificadas en diversos reportes de investigación nacional e internacional de los últimos años, el grupo de investigadores concluye de manera general:

- La expresión "educación inclusiva" es cada vez más frecuente en los planes de estudio de las instituciones de educación superior alrededor del mundo que buscan formar docentes en la carrera de EE.

- En Europa, Estados Unidos y América Latina, con excepción de Chile, no se ha logrado establecer un perfil profesional oficial para la EE. Chile estableció unos estándares para realizar la evaluación del desempeño del grupo de profesionales en la disciplina, que sirven como referente para el diseño curricular y además para establecer requisitos de selección y contratación docente (Godoy, Otondo y Rey, 2016).

- Las carreras de EE diseñan su perfil particular mediante el planteamiento de grandes rasgos en atención al criterio de sus expertos, lo que se refleja en sus planes de estudios (Meléndez, 2020).

- Los perfiles de egreso no reflejan el desempeño profesional; tienen limitaciones en cuanto a la contextualización de estos.

\section{REFERENTES TEÓRICOS}

En Costa Rica la educación inclusiva empezó a dibujarse desde los años 70, en busca de la superación de la educación segregada y para convertir los sitios educativos en espacios participativos de representación social y cultural para todos. En concordancia con las tendencias mundiales durante la década de los 80 empezaron a reportarse acciones donde personas con discapacidad visual participaban en entornos menos restrictivos. Ya para la década de los 90 las residencias segregadas en centros educativos exclusivos para personas en situación de discapacidad visual empezaron a cerrarse y la población infantil pudo volver a sus hogares y comunidades (Meléndez, 2005, 2020; MEP, 2016 y OCDE, 2017).

De los años 2000 en adelante podría decirse que el camino hacia la educación inclusiva ha sido congruente con el modelo social, dando más énfasis a aspectos relacionados con los Derechos Humanos y dejando paulatinamente las tendencias rehabilitadoras, tanto para las personas con discapacidad como para aquellas que por diferentes situaciones no han podido asumir su derecho a educarse.

En Costa Rica, la EE ha significado un apoyo e impulso para el establecimiento de la educación inclusiva. En el país, la educación general básica, que va desde la primera infancia a tercer ciclo, se complementa con la diversificada y la especial, en una relación dialéctica que requiere nuevas definiciones, desde las raíces, de los roles de cada profesión docente. El conjunto se configura así cada vez más como un único sistema educativo. Todos los niveles educativos son obligatorios y costeados por el Estado.

Bajo esta coyuntura, el año 2014 vio nacer la Comisión Interinstitucional para el Análisis Epistemológico de la EE en Costa Rica, integrada por varias universidades públicas: Universidad Estatal a Distancia (UNED), Universidad de Costa Rica (UCR) y Universidad Nacional (UNA), y otras instancias atinentes como el Centro Nacional de Recursos para la Educación Inclusiva (CENAREC), el Sistema Nacional de Acreditación de la Educación Superior (SINAES) y el Departamento de EE del Ministerio de Educación Pública (hoy Departamento de Apoyos Educativos para el Estudiantado con Discapacidad del MEP). Dicha comisión, como parte de sus acciones iniciales, organizó en el año 2016 la I Jornada de Análisis 
Epistemológico de la EE en Costa Rica, en la cual se construyó de manera cooperativa, a partir de la visión de educación inclusiva y del enfoque de derechos, la definición del profesional de esta rama, como la persona

profesional capaz de construir, organizar, liderar, proponer y practicar, colaborativamente, estrategias pedagógicas para atender a la diversidad, y de catapultar la superación de barreras para el aprendizaje y la participación del estudiantado, incluidas las personas que requieren apoyos extensos y generalizados; así como de mantener un conocimiento actualizado mediante la investigación permanente y la reflexión de su propia práctica (Castillo et al. 2018, p. 29).

Lo anterior sirvió como marco de partida para la siguiente acción que asumiría la comisión, dirigida a dilucidar cuál debía ser el perfil de ese docente capaz de enfrentar los nuevos retos de la EE costarricense en entornos inclusivos. La formación profesional en el país se desarrolla a nivel universitario.

Es importante señalar que la comisión interinstitucional continuó fortaleciéndose y a partir del año 2018 tomó el nombre de Observatorio Nacional de la Educación Inclusiva (ONEI); se integraron el Colegio de Licenciados y Profesores en Letras, Filosofía Ciencias y Artes de Costa Rica (COLYPRO), se invitó a las universidades privadas del país que brindaban la carrera de EE para que formaran parte del ONEI, y se incorporó de manera activa a la Universidad Católica de Costa Rica. Actualmente el Observatorio está integrado por 14 personas con experiencia en el campo de la docencia universitaria y la investigación, con formaciones en áreas diversas como EE, educación general básica, evaluación educativa, administración educativa, psicología, psicopedagogía, sociología, orientación y trabajo social.

Distintas evaluaciones de la educación costarricense - manifiestas en el informe de la Organización para la Cooperación y el Desarrollo Económicos (OCDE, 2017), en el Sexto Informe del Estado de la Educación (PEN, 2017) y en las conclusiones del Foro "Diálogos sobre los desafíos de la educación OCDE-MEPPrograma Estado de la Nación (PEN)", que se llevó a cabo en octubre del 2017- señalan algunas deficiencias del sistema educativo nacional, entre ellas, la ausencia de perfiles profesionales en los docentes que atienden los diversos servicios y niveles. Esto se sumó a las razones que ya acumulaba el ONEI para iniciar con la tarea investigativa para diseñar y validar el "Perfil del profesional de la EE costarricense desde un enfoque inclusivo" (Meléndez et al., 2020).

$\mathrm{Al}$ respecto, Meléndez (2020) señala la importancia de asumir la EE de forma integral, a la vez que debe abarcarse desde un enfoque inclusivo "tanto su concepción ontológica, sus principios epistemológicos y éticos, hasta la forma en que se disponen los servicios (...) en cada país y, por supuesto, las ofertas de formación” (p. 269). Esto se une al compromiso con el objetivo 4 de desarrollo sostenible asumido por Costa Rica en su política educativa, que aboga por una educación inclusiva de calidad durante toda la vida (UNESCO, 2015 y MEP, 2016).

El equipo de investigadores comprende a la educación inclusiva como un derecho humano fundamental para la superación de las personas desde su propia diversidad, en el seno de su familia y comunidad y en beneficio integral de la sociedad costarricense. Se fundamenta en la equidad, la participación y el aprendizaje con un currículum educativo flexible, efectivo y de calidad, que se operacionaliza mediante el diseño universal de los aprendizajes en todos los niveles y modalidades del sistema educativo. (CENAREC, 2017; Asamblea Legislativa, 2018 y UNESCO, 2021). La educación especial se constituye en una de las disciplinas que brinda apoyos diversos a la gestión de los procesos de enseñanza y aprendizaje.

\section{METODOLOGÍA}

Ante la ausencia de un perfil profesional de la EE de Costa Rica, así como de teoría local al respecto, el ONEI se dio a la tarea de su construcción mediante un proceso de investigación desde los aportes del método mixto y de orden secuencial, el cual Villalobos (2017, p. 365) plantea como "un conjunto de procesos sistemáticos que tratan de integrar métodos de investigación (...) para la recolección de datos 
cualitativos y cuantitativos respectivamente, de forma diacrónica o sincrónica, para obtener inferencias y lograr un mayor conocimiento del fenómeno social".

El objetivo orientador del trabajo fue el diseño del perfil profesional de la persona educadora, al cual se otorga validez a partir de una construcción participativa. El interés del presente documento es compartir ese transitar.

Como parte de este proceso, el equipo de investigación utilizó como metodología la estrategia secuencial exploratoria de Creswell (2003), asumida desde una posición inductiva, abierta, flexible, inconclusa, integral y sistémica, que permitió alternar ambos métodos, cualitativo y cuantitativo, en la indagación del fenómeno que se planteó. Desde el punto de vista metodológico se interpreta que la construcción del perfil profesional en EE se convierte en un potencial recurso en los procesos de formación continua y en la gestión de los servicios de apoyo en los contextos de la educación inclusiva costarricense.

La investigación se desarrolló a lo largo de cuatro etapas y sus resultados fueron objeto de diferentes procesos de validación y reconstrucción. En la etapa final se concluye con la definición de las funciones que integran el perfil profesional definitivo de la EE en Costa Rica. Se utilizó frecuentemente el criterio de personas expertas, lo que permitió el análisis comparativo desde diferentes visiones disciplinares y el establecimiento de un perfil profesional mediante el consenso de las personas participantes (Benavides y Gómez-Restrepo, 2005). En todo momento se siguieron las normas éticas para la participación de las personas de forma consentida e informada. La figura 1 resume las fases y etapas metodológicas desarrolladas.

FIGURA 1

Esquema resumen de la metodología utilizada para la elaboración del perfil profesional de la EE, Costa Rica

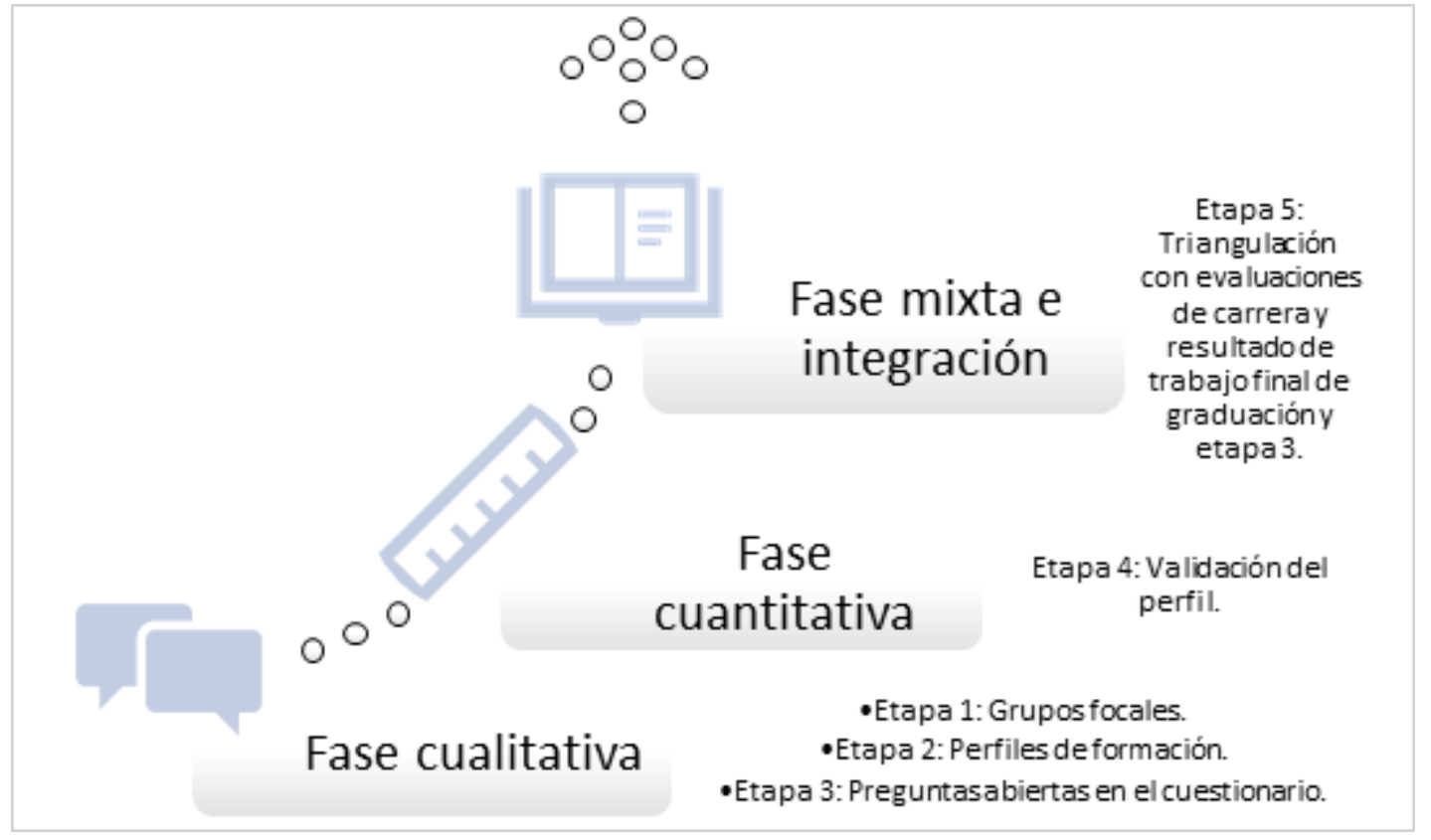

Elaboración propia, 2020

\subsection{Descripción de las fases y etapas de investigación}

A continuación se describen las fases realizadas de acuerdo con sus etapas y se refieren las personas participantes, técnicas e instrumentos utilizados, así como los procedimientos para el análisis de la información. 


\subsection{Fase cualitativa}

La fase cualitativa de este estudio comprendió tres etapas en la secuencialidad caracterizada por el tipo mixto de investigación que se siguió. Se asumió como método la fenomenología, ya que resultó de interés identificar las esencias interpretadas y vividas por las personas participantes en el estudio (Creswell, 2003). La primera etapa tuvo que ver con el acercamiento a la temática mediante grupos focales de profesionales de la EE, dada la escasez de material teórico relacionado con la construcción de perfiles profesionales en Costa Rica, la cual se nota más agudamente en el campo de la educación. Y, tal cual se describe también en este apartado, la segunda etapa tuvo que ver con el análisis cualitativo de perfiles de formación en el área, y la tercera, con el análisis de respuestas a preguntas abiertas incluidas en los cuestionarios aplicados, los cuales se describen en la fase cuantitativa.

\subsubsection{Etapa 1}

Esta fase se realizó a mediados del 2018; participaron 37 mujeres profesoras universitarias de la carrera de EE de tres universidades públicas (UCR, UNA, UNED) y una privada (Universidad Latina, en ese momento integraba el ONEI). Las profesoras contaban con un rango de experiencia en los servicios educativos de 7 a 25 años, 16 profesoras participaron en grupos focales y 21 de la UNED, por su modalidad a distancia, respondieron un cuestionario en línea que contenía preguntas equivalentes a las presentadas a los grupos focales. La participación únicamente de mujeres se debe a razones contextuales, ya que la carrera en el país es principalmente femenina; su participación en esta actividad fue voluntaria.

En esta oportunidad, las profesoras universitarias reflexionaron alrededor de las siguientes preguntas orientadoras:

1. ¿Cuáles estrategias requiere el profesional en EE para desarrollar una mediación pedagógica orientada hacia la diversidad?

2. ¿Cuáles estrategias recomiendan para trabajar el acercamiento con la familia y la comunidad en el marco de la educación inclusiva?

3. ¿Cómo se evidencia en los procesos de educación inclusiva el profesorado investigador reflexivo de EE?

4. ¿En cuáles temáticas considera que es necesaria la formación y actualización del profesorado de EE para desarrollar procesos de educación inclusiva?

5. ¿A partir de qué criterios se definen las estrategias de mediación pedagógica en EE?

La información recuperada de esta fase se analizó con el programa Atlas-Tiं, a partir de categorías inductivas referidas a enfoques, investigación de la práctica docente, estrategias de enseñanza, familias y el estudiantado.

\subsubsection{Etapa 2}

Entre los inicios del año 2018 y 2019 se realizó un análisis documental de los perfiles de egreso de las carreras de EE de universidades nacionales e internacionales. Se utilizaron 12 perfiles, de los cuales tres correspondían al continente europeo (Martínez - Izaguirre, Villardón y Yániz, 2013; Almenta y Leiva, 2018), uno a Sur América (Ortiz, 2007), y ocho costarricenses, tres de universidades estatales UCR, UNA, UNED y cinco de privadas, a saber, Universidad San Isidro Labrador, Universidad Florencio del Castillo, Universidad Católica, Universidad de la Ciencias y el Arte y Universidad Latina de Costa Rica (Alfaro y Alvarado, 2018). 
Como producto de la fase cualitativa se obtiene un listado de 98 funciones distribuidas en 3 dimensiones: conceptual, actitudinal y procedimental y 7 áreas de competencia: 1. Enfoques teóricos, metodológicos, políticos y legales; 2. Investigación y evaluación de la práctica; 3. Desarrollo Humano; 4. Mediación pedagógica; 5. Asesoramiento: familiar, comunitario, institucional; 6. Gestión: administración, desarrollo de proyectos y 7. Redes de apoyo familiar, comunitario, interprofesional.

\subsubsection{Etapa 3}

Con las 98 funciones se creó una serie de formularios de preguntas abiertas y cerradas para validarlas desde su claridad y pertinencia tanto en las dimensiones como las áreas de competencia propuestas. Para esta etapa, con el fin de analizar las respuestas abiertas que dio el profesorado al final de cada una de las secciones de los cuestionarios, se aplicó el programa Atlas Ti; para ello se conformó una subcomisión que, de forma deductiva, organizó en categorías los textos producidos, considerándolos como emergentes y respetando la naturaleza multicíclica, en espiral y flexible de la investigación cualitativa que señala Gurdián (2007). Así se construyeron las categorías: actitudes del profesorado, evaluación docente, condiciones laborales y capacitación docente. Si bien no todas estas categorías aludían directamente a funciones del perfil, permitieron reconstruir algunas de las condiciones del contexto laboral docente para enmarcar las funciones en una realidad congruente.

\subsubsection{Fase cuantitativa}

La fase cuantitativa se caracterizó por seguir un método descriptivo (Villalobos, 2017), pues con mediciones se buscó establecer el contenido del perfil de forma sistemática, tanto en el diseño como en el análisis y establecimiento de resultados mediante el uso de paquetes estadísticos y técnicas para el aseguramiento de las evidencias de validez de la información obtenida, tal como se describe en la etapa 4.

\subsubsection{Etapa 4}

Como lo indican Bernal y Arango (2017, p.55), el uso del cuestionario en la investigación social permite la recolección de "gran cantidad de datos sobre, actitudes, intereses, opiniones, conocimiento, comportamiento (pasado, presente y esperado), etc. Así como los datos de clasificación relativos a las medidas de carácter demográfico y socioeconómico, de forma relativamente ágil y económica”, por lo que se diseñó un cuestionario madre $(\mathrm{Q})$ con las áreas de competencia y sus respectivas dimensiones. Para evitar el cansancio de las personas participantes se organizó en cinco versiones de contenido indagatorio equivalente, como se detalla en la Tabla 1, distribuyendo el contenido del cuestionario Q. 
TABLA 1

Áreas de competencia asignadas a cada cuestionario

\begin{tabular}{ll}
\hline Áreas de competencia & Cuestionario \\
\hline $\begin{array}{l}\text { 1. Enfoques teóricos, metodológicos, políticos y legales } \\
\text { 2. Investigación y evaluación de la práctica }\end{array}$ & Q1 \\
$\begin{array}{l}\text { 3. Mediación pedagógica } \\
\text { 4. Desarrollo humano }\end{array}$ & Q2 \\
$\begin{array}{l}\text { 5. Redes de apoyo familiar, comunitario, interprofesional } \\
\text { 6. Gestión: administración, desarrollo de proyectos }\end{array}$ & Q3 \\
\begin{tabular}{l} 
7. Asesoramiento: familiar, comunitaria, institucional \\
\hline TOTAL
\end{tabular} & Q4/ Q5 \\
\hline
\end{tabular}

Tomado de Meléndez et al. (2020, p.33).

Los cuestionarios Q1, Q2, Q3 se enviaron a 3500 docentes en ejercicio de EE que se encontraban en las bases de datos del COLYPRO al momento del estudio, por lo tanto, la muestra fue de tipo no probabilística intencionada; el total de docentes se distribuyó por provincia, según el lugar donde se encontraba el centro educativo en el cual estaba nombrado. Los 3 cuestionarios se enviaron a un número igual de docentes por provincia.

Se recuperaron un total de 713 cuestionarios completos (Q1n=196, Q2n=241, Q3n=276), lo que representa un $20 \%$ de la población contactada a través de correo electrónico, para ello se estimuló la participación docente a través del sorteo de incentivos para aquellas personas que finalizan el cuestionario autoadministrado a través de la plataforma online dispuesta para ese fin (Survey Monkey ${ }^{\circ}$, licencia autorizada del COLYPRO).

La edad mínima de los participantes fue de 23 años, mientras que la máxima fue de 61 años, siendo el promedio los 40 años. En su mayoría contaban con el grado de licenciatura. La mayor cantidad de docentes que completaron el cuestionario fue de la provincia de San José (213 personas), seguido por Alajuela y Guanacaste ( 55 personas).

La versión del cuestionario nombrada Q4 incorporaba la totalidad de funciones, y se aplicó a 60 personas asesoras nacionales de EE y de otras áreas de las Ciencias de la Educación del MEP, así como a seis personas administradoras de centros educativos de EE y dos de Educación General Básica a nivel nacional. La edad media de esta población fue de 46,5 años. La mayoría de las personas participantes ostentaba el grado de maestría.

El cuestionario Q5 se aplicó a las personas especialistas internacionales que han sido evaluadores externos para el SINAES de las carreras de EE. Se contó con el apoyo invaluable de Libia Vélez Latorre, de la Universidad Pedagógica Nacional de Colombia; Jorge Iván Correa Alzate del Tecnológico de Antioquia, Colombia; José Juan Carrión Martínez de la Universidad de Almería, España, y José Gabriel Padín, consultor independiente, Estados Unidos, todos con el grado académico de doctor. De estos, tres cuestionarios se recobraron completos.

Cada cuestionario constaba de dos secciones, una de datos sociodemográficos básicos como la edad y el grado académico y luego el listado de funciones distribuidas en módulos por áreas de competencia. Cada módulo estaba compuesto por tres matrices, una correspondiente a las funciones en la dimensión conceptual, procedimental y actitudinal respectivamente. Cada matriz tenía el listado de sus funciones, las que se valoraron a través de los tres criterios establecidos, los cuales estaban compuestos por sus respectivos indicadores. 
Mediante este mecanismo, el profesional en EE podía juzgar la claridad, importancia y la pertinencia de cada función en la dimensión correspondiente, y en el área de competencia en la cual la función estaba asignada.

Con el aporte metodológico de Tineo, 2012; Martínez-Izaguirre, Villardón y Yániz, 2013, y Alfaro y Alvarado, 2018, cada función incluida en el cuestionario se valoró a partir de los siguientes criterios y opciones de respuesta:

- Claridad de la función: la función está redactada de manera comprensible. ( 1 =No está claro, 2=Está claro)

- Importancia para con el contexto educativo: la función es necesaria para el contexto educativo. Se utilizó una escala de tipo Likert ( $1=$ Nada importante, $2=$ Poco importante, $3=$ Importante, 4=Muy importante), sin embargo, en el análisis de resultados se optó por dicotomizar las opciones de respuesta para tener mayor fuerza, por lo tanto, los códigos 1 y 2 se recodificaron como " $1=$ No importante" y los códigos 3 y 4 como " 2 = Importante", la opción de estos ítems dicotómicos también favoreció la aplicación del alfa de Cronbach (Hernández-Sampieri, Fernández Collado y Baptista Lucio, 2014) para medir la consistencia interna de cada uno de los instrumentos.

- Pertinencia en la dimensión: la función pertenece a la dimensión asignada. ( $1=$ No pertinente, $2=$ Pertinente)

Al finalizar la aplicación fueron extraídas de la plataforma Survey Monkey ${ }^{\circledR}$ las gráficas de los resultados del análisis de frecuencia y las respuestas a los ítems abiertos.

Para cada cuestionario se generó una base de datos propia y se procesó con el programa informático para análisis estadísticos Statistical Package for the Social Sciences (SPSS v.21) de manera independiente utilizando datos descriptivos, de dispersión de datos, unidimensionalidad de los factores, antecedidos por la prueba de adecuación de Kaiser-Meyer Olkin (KMO) y el valor del Alfa de Cronbach (López-Aguado y Gutiérrez-Provecho, 2019). Para cada uno de los criterios de claridad, importancia y pertinencia se trabajó con el valor del promedio de las puntuaciones asignadas.

Finalmente, para concluir la fase cuantitativa se aplicó el análisis factorial exploratorio, que tuvo como objetivo descubrir variables no observadas (constructos) que explican por qué algunos ítems se relacionan más con unos que con otros (varianza compartida). Morales (2011, p. 5) indica que "examinando el contenido conceptual de los ítems que pertenecen al mismo factor podemos comprender qué factores [o constructos] subyacentes explican las correlaciones entre los ítems".

\subsubsection{Fase mixta e integración}

La quinta y última etapa de análisis y validación se hace mediante tres actividades que consolidan los resultados y hallazgos, así como la rigurosidad científica del estudio y el consenso, que retrata la participación tanto de las fuentes teóricas como de las voces de las personas informantes validadoras que se involucraron con sumo interés en la pesquisa realizada.

\subsubsection{Etapa 5}

Las funciones resultantes fueron sometidas a un proceso de triangulación mediante el análisis de las personas expertas integrantes del ONEI, quienes trabajaron por subgrupos para conjuntar, de manera organizada y según las dimensiones y áreas de competencia preestablecidas, las funciones derivadas de cada cuestionario en un solo listado, que venía a constituir el perfil profesional final. 
Además, en este análisis cualitativo se triangularon las funciones con los resultados del análisis de las fortalezas y las recomendaciones encontradas en la Evaluación Externa y evaluación de Informes de Logro del Proyecto Especial de Mejoras de carreras nacionales de EE acreditadas por parte del SINAES (Zúñiga, 2020). Los datos correspondieron a cinco carreras de EE acreditadas en Costa Rica, desde el año 2011 al año 2019. En total fueron analizados 8 informes de Evaluación Externa y 2 informes de Logro del Proyecto Especial de Mejoras, dado que durante los años citados algunas de estas carreras se reacreditaron. Esta actividad fue muy valiosa, en tanto dichas fortalezas y recomendaciones se relacionaban en buena medida con el deber ser del profesional en formación, lo que alude directamente a la preparación de calidad para el ejercicio de funciones docentes.

También se utilizaron los hallazgos de Cordero et al. (2019), quienes estudiaron el aporte de las personas de las carreras de Educación Preescolar y Educación Especial al abordar la diversidad en su quehacer pedagógico.

\section{ANÁLISIS DE RESULTADOS}

A continuación, se explica el modo en que se obtuvieron resultados y hallazgos de cada una de las fases metodológicas que se siguieron para la elaboración del perfil profesional de la disciplina de EE. Coherente con el método mixto que se asumió en esta investigación, este apartado se ha organizado en las tres fases de recopilación y construcción de evidencias para garantizar la rigurosidad científica; a saber, fase de trabajo cualitativo, cuantitativo y de articulación e integración de aportes propios de la fase mixta.

\subsection{Fase Cualitativa}

En el trabajo con los grupos focales de docentes de las universidades estatales y privadas, surgió un conjunto de categorías referidas a aspectos axiológicos y actitudinales de la práctica docente, mediación pedagógica y estrategias educativas, vinculación de la familia y la comunidad e investigación, como se ilustra en la Tabla 2. 
TABLA 2

Ejemplo de categorías utilizadas para la elaboración del perfil profesional de la EE, etapa 1. Costa Rica.

\begin{tabular}{|c|c|c|}
\hline $\begin{array}{l}\text { Investigación } \\
\text { Códigos (11): }\end{array}$ & $\begin{array}{l}\text { Familia y comunidad } \\
\text { Códigos (12): }\end{array}$ & $\begin{array}{l}\text { Educando } \\
\text { Códigos (14): }\end{array}$ \\
\hline Investigador: & Familia y comunidad: & Educando: Autonomía- \\
\hline Actualizarse $\{4-1\}$ & Actividades-actos cívicos- & independencia $\{1-1\}$ \\
\hline Investigador: Conocer & presentaciones- & Educando: \\
\hline programas $\{2-1\}$ & celebraciones $\{6-2\}$ & Características edad $\{3-$ \\
\hline Investigador: Construye & Familia y comunidad: & $2\}$ \\
\hline metodologia-estrategias & Empoderar a familia $\{3-1\}$ & Educando: \\
\hline$\{8-2\}$ & Familia y comunidad: & Competencias para la \\
\hline Investigador: Crea & Entrevistas- encuestas $\{4-2\}$ & vida $\{6-11\}$ \\
\hline $\begin{array}{l}\text { instrumentos-registra- } \\
\text { recolecta datos }\{7-2\}\end{array}$ & $\begin{array}{l}\text { Familia y comunidad: } \\
\text { Estrateqias concretas con }\end{array}$ & $\begin{array}{l}\text { Educando: Crianza } \\
\text { hogar }\{3-2\}\end{array}$ \\
\hline Investigador: Evaluación- & familias $\{8-2\}$ & Educando: Enseñar \\
\hline valoración integral $\{13-9\}$ & Familia y comunidad: & convivir $\{2-1\}$ \\
\hline $\begin{array}{l}\text { Investigador: Genera } \\
\text { hipótesis y preguntas }\{2- \\
2\}\end{array}$ & $\begin{array}{l}\text { Familia con estereotipos }\{2- \\
2\} \\
\text { Familia v comunidad: }\end{array}$ & $\begin{array}{l}\text { Educando: Enseñar } \\
\text { habilidades blandas }\{3- \\
1\}\end{array}$ \\
\hline $\begin{array}{l}\text { Investigador: Genera } \\
\text { resultados-propuestas- }\end{array}$ & $\begin{array}{l}\text { Información-comunicación } \\
\{9-1\}\end{array}$ & $\begin{array}{l}\text { Educando: Enseñar } \\
\text { respeto }\{3-1\}\end{array}$ \\
\hline acciones $\{8-5\}$ & Familia y comunidad: & Educando: Enseñar \\
\hline Investigador: & Involucrar a la comunidad & tolerante $\{1-2\}$ \\
\hline Observación $\{5-1\}$ & $\{3-3\}$ & Educando: Enseñar \\
\hline Investigador: Reflexiona & Familia y comunidad: & valores $\{1-3\}$ \\
\hline $\begin{array}{l}\text { sobre su práctica }\{12-2\} \\
\text { Investigador: }\end{array}$ & $\begin{array}{l}\text { Involucrar familia en el } \\
\text { proceso educativo }\{6-7\}\end{array}$ & $\begin{array}{l}\text { Educando: poner } \\
\text { atención }\{1-1\}\end{array}$ \\
\hline $\begin{array}{l}\text { Sistematiza-analiza } \\
\text { datos }\{7-2\}\end{array}$ & $\begin{array}{l}\text { Familia y comunidad: Redes } \\
\text { de apoyo }\{5-4\}\end{array}$ & $\begin{array}{l}\text { Educando: seguir } \\
\text { instrucciones }\{3-1\}\end{array}$ \\
\hline Investigador: Sustenta en & Familia y comunidad: & Educando: Ser \\
\hline marco teórico $\{5-4\}$ & Talleres-charlas- & ordenado $\{1-1\}$ \\
\hline & capacitaciones $\{15-5\}$ & Educando: Trabajar con \\
\hline & Familia y comunidad: & los talentosos $\{1-1\}$ \\
\hline & $\begin{array}{l}\text { Trabajo colaborativo con } \\
\text { familia }\{5-1\}\end{array}$ & $\begin{array}{l}\text { Educando: Trabajo } \\
\text { colaborativo- }\end{array}$ \\
\hline & $\begin{array}{l}\text { Familia y comunidad: Visitas } \\
\text { familia y comunidad }\{6-2\}\end{array}$ & $\begin{array}{l}\text { comunicación con el } \\
\text { otro }\{3-1\}\end{array}$ \\
\hline
\end{tabular}

Tomado de Meléndez et al. (2020, p. 89).

Del análisis inductivo de los perfiles de formación profesional docente de las carreras se identificaron y sistematizaron las primeras categorías, que luego pasarían a ser áreas de competencia, en tanto se intuía que dentro de estas deberían ser clasificadas en adelante las funciones que se lograra identificar en el proceso:

- Enfoques teóricos, ideológicos políticos y legales.

- Desarrollo humano.

- Mediación pedagógica: Esta área en particular incluyó aspectos relacionados con evaluación inicial y de los aprendizajes, planificación, estrategias de mediación a partir de los referentes del Diseño 
Universal para el Aprendizaje, el aprendizaje colaborativo y el manejo conductual positivo; otro aspecto fue el diseño de apoyos humanos y tecnológicos.

- Redes de apoyo: Comprendidas a partir de la vinculación de la familia, la comunidad y los diferentes grupos profesionales en los procesos educativos del estudiantado.

- Gestión: Incluye la toma de decisiones administrativas y el desarrollo de proyectos institucionales y comunales.

- Asesoramiento: Cómo es el rol que asume el profesional de EE para acompañar la toma de decisiones a nivel institucional-educativo, familiar y comunal.

- Evaluación de la propia práctica/Investigación: En esta primera aproximación el grupo de investigadores observa que es necesario profundizar en la reflexión de la categoría vinculada con la evaluación e investigación de la propia práctica docente; además, se deben articular los ejes transversales del modelo social de la discapacidad, a la vez que la definición del profesional en EE obtenida durante las jornadas.

En la etapa 2 se encontró que hay categorías similares a las identificadas en la etapa 1, tales como la mediación pedagógica y la participación de la familia y la comunidad, lo que permitió tener evidencias de la importancia de estas, debido a la saturación presentada en los diferentes análisis.

Una vez determinado el conjunto de áreas de competencia, se procedió a revisar la compilación de funciones derivadas de las fuentes consultadas hasta ese momento, con el fin de clasificarlas en cada una de las áreas según el criterio de los investigadores expertos del equipo ONEI.

Finalmente se definió un conjunto de 98 funciones clasificadas en siete grandes áreas de competencia, que detallaban el perfil profesional del profesorado de EE que sería sometido al proceso de validación. Cabe agregar que las áreas de competencia, a su vez, tenían las dimensiones conceptual, actitudinal y procedimental, establecidas oficialmente, por el CONARE y el CONESUP, para el diseño de perfiles de formación de la educación superior costarricense, tal cual se observa en la siguiente tabla. 
TABLA 3

Perfil inicial: cantidad de funciones según áreas de competencia y dimensiones de la educación superior

\begin{tabular}{|c|c|c|c|c|}
\hline Áreas de competencia & $\begin{array}{l}\text { Dimensión } \\
\text { conceptual }\end{array}$ & $\begin{array}{l}\text { Dimensión } \\
\text { actitudinal }\end{array}$ & $\begin{array}{l}\text { Dimensión } \\
\text { procedimental }\end{array}$ & $\begin{array}{l}\text { Total } \\
\text { por } \\
\text { área }\end{array}$ \\
\hline $\begin{array}{l}\text { F1. Enfoques teóricos, } \\
\text { metodológicos, } \\
\text { políticos y legales }\end{array}$ & 6 & 6 & 6 & 18 \\
\hline $\begin{array}{l}\text { 2. Investigación y } \\
\text { evaluación de la } \\
\text { práctica }\end{array}$ & 5 & 2 & 5 & 12 \\
\hline $\begin{array}{l}\text { 3. Mediación } \\
\text { pedagógica }\end{array}$ & 11 & 9 & 10 & 30 \\
\hline 4. Desarrollo humano & 6 & 5 & 4 & 15 \\
\hline $\begin{array}{l}\text { 5. Redes de apoyo } \\
\text { familiar, comunitario, } \\
\text { interprofesional }\end{array}$ & 7 & 2 & 4 & 13 \\
\hline $\begin{array}{l}\text { 6. Gestión: } \\
\text { administración, } \\
\text { desarrollo de proyectos }\end{array}$ & 2 & 1 & 3 & 6 \\
\hline $\begin{array}{l}\text { 7. Asesoramiento: } \\
\text { familiar, comunitaria, } \\
\text { institucional }\end{array}$ & 1 & 2 & 1 & 4 \\
\hline TOTAL & 27 & 38 & 33 & \\
\hline
\end{tabular}

\subsection{Fase Cuantitativa}

Debido a la cantidad de las funciones, someter todas a revisión por cada docente en ejercicio de la muestra podía resultar agotador, por lo que se corría el riesgo de muerte muestral. Entonces, para ser sometido a la validación de los docentes, el listado de funciones se subdividió en tres partes equivalentes y cada una de estas constituyó un instrumento, el fragmento asignado de funciones distribuidas en las áreas de competencia y las dimensiones en subdivisiones previamente determinadas. Adicionalmente, se aplicaron y procesaron dos cuestionarios que contenían la totalidad de las funciones a las autoridades del MEP a nivel nacional y regional, así como a las personas expertas en la disciplina a nivel internacional que participaron en la validación de áreas y funciones del perfil.

A pesar de que inicialmente se había considerado una escala Likert de cuatro puntos para la medición del criterios de importancia, en una sesión con las personas integrantes del ONEI se concluyó que los valores intermedios no permitían la toma de decisiones para lograr depurar el perfil, por lo que se acordó dicotomizar los resultados en dos nuevas categorías de análisis extremas -referidas al cumplimiento (2) o al no cumplimiento (1) del criterio establecido- y se procedió a la recodificación de las variables. 
Para la toma de decisiones en la revisión de las funciones se trabajó con los tres criterios de pertinencia, importancia y claridad, el promedio y su respectiva desviación estándar. A su vez se calculó la media y la desviación estándar del total de funciones.

Con el fin de lograr una mayor depuración de cada una de las funciones, el grupo de investigadores estableció de forma arbitraria que aquellas funciones que obtuvieran 0,03 puntos por debajo de la media y de la desviación estándar del total de funciones por criterio requerirían de una valoración integral para decidir si se eliminaba, modificaba o se mantenía como se había considerado inicialmente.

Otros insumos para la selección de las funciones finales del perfil fueron los resultados obtenidos del análisis factorial exploratorio (AFE) y el valor del alfa de Cronbach. En el caso del AFE se analizaron aquellas funciones que no se encontraban dentro de los tres primeros factores de la matriz de factores rotados con el objetivo de determinar la unidimensionalidad y la pertinencia de las funciones. También se utilizó como criterio la revisión de las funciones que podían aumentar el valor de la consistencia interna de las respuestas y eliminar aquellas que no alcanzaran valores adecuados (menores a 0,3 ). Toda la información previamente descrita se organizó en tres cuadernillos que incluían el cuestionario respectivo de docentes, los resultados de los representantes del MEP y las personas expertas internacionales.

Con todos los insumos anteriores, cada subgrupo de trabajo utilizó la plantilla que se observa en la Tabla 4 como ejemplo, la cual permitió establecer si cada función requería ajustes en su redacción, era pertinente en toda su propuesta, o, en caso contrario, debía ser descartada por no cumplir con ninguno de los aspectos de rigurosidad establecidos.

En la Tabla 4 se puede observar, por ejemplo, que la función 4 de la dimensión conceptual (C4), presentaba valores menores al 0,03 en la media general de todas las funciones, así como en las desviaciones estándar de los tres criterios establecidos, tanto desde la opinión docente, como de los representantes del MEP; además, si bien a nivel teórico pertenecía al factor uno, en los resultados del análisis de factores se ubicó en el factor 4 . Por su parte, las personas expertas internacionales valoraron que la función era clara, importante y pertinente. Con todo este conjunto de insumos el grupo de investigadores decidió que esta función se integrara a las funciones $\mathrm{C} 3$ y C5, como se detalla en la Tabla 4.

En el caso de la función 20 de la dimensión actitudinal representada en la Tabla 4, se puede observar que, de acuerdo con el criterio docente, de los representantes del MEP y de las personas expertas internacionales, cumplió con los criterios de claridad, importancia y pertinencia, por lo que el grupo de investigadores decidió mantenerla como se presentaba inicialmente. 
TABLA 4

Ejemplo de matriz de cuadernillo para revisión de cada una de las funciones de las dimensiones conceptual, procedimental y actitudinal.

\begin{tabular}{|c|c|c|c|c|c|c|c|}
\hline \multirow[b]{2}{*}{ Función } & \multicolumn{2}{|c|}{$\begin{array}{l}\text { Criterio de } \\
\text { docentes }\end{array}$} & \multicolumn{2}{|c|}{$\begin{array}{l}\text { Representantes del } \\
\text { MEP }\end{array}$} & \multirow{2}{*}{$\begin{array}{l}\begin{array}{l}\text { Persona } \\
\text { expertas } \\
\text { internaci } \\
\text { onales }\end{array} \\
\text { aloraciōn } \\
\text { realizada } \\
\end{array}$} & \multirow[t]{2}{*}{$\begin{array}{l}\text { Factor en } \\
\text { que se ubica }\end{array}$} & \multirow{2}{*}{$\begin{array}{l}\text { Observaciones del grupo revisor } \\
\text { Si se modifica: Anotar los } \\
\text { cambios que se realizan }\end{array}$} \\
\hline & $\bar{x}$ & $\sigma$ & $\bar{x}$ & & & & \\
\hline $\begin{array}{l}\text { C4 } \\
\text { Identificar el } \\
\text { propósito y } \\
\text { formas de uso } \\
\text { de } \\
\text { herramientas } \\
\text { evaluadoras } \\
\text { disponibles } \\
\text { tanto en } \\
\text { medios } \\
\text { digitales como } \\
\text { en fisico. }\end{array}$ & $\begin{array}{l}\text { Clarida } \\
\text { d: } 1,80 \\
<1,87^{*} \\
\text { Importa } \\
\text { ncia } \\
1,96< \\
1,98^{*} \\
\text { Pertine } \\
\text { ncia: } \\
1,95<1, \\
98^{*}\end{array}$ & $\begin{array}{l}\text { Clarida } \\
\text { d: } \\
0,40>0, \\
30^{*} \\
\text { Importa } \\
\text { ncia: } \\
0,20>0 \text {, } \\
13^{*} \\
\text { Pertine } \\
\text { ncia: } \\
0,23>0 \text {, } \\
14^{*}\end{array}$ & $\begin{array}{l}\text { Claridad. } \\
1,90<1,9 \\
3 \\
\text { Importan } \\
\text { cia: } \\
1,98=1,9 \\
8 \\
\text { Pertinen } \\
\text { cia: } \\
1,98=1,9 \\
8\end{array}$ & $\begin{array}{l}\text { Clarida } \\
\text { d: } \\
0,30>0, \\
25^{*} \\
\text { Importa } \\
\text { ncia: } 0,1 \\
3=0,13^{*} \\
\text { Pertine } \\
\text { ncia: } \\
0,13<0 \text {, } \\
14^{*}\end{array}$ & $\begin{array}{l}\text { La } \\
\text { función } \\
\text { es } \\
\text { clara, } \\
\text { importa } \\
\text { nte y } \\
\text { pertinen } \\
\text { te. }\end{array}$ & $F 4=590$ & $\begin{array}{l}\text { El grupo considera, que el item } \\
\text { se debe integrar con los items } \\
\text { F1C3 y F1C5 obteniendo: } \\
\text { Reconocerla importancia del } \\
\text { contexto del estudiantado para el } \\
\text { diseño de apoyos educativos con } \\
\text { técnicas e instrumentos } \\
\text { evaluativos tanto digtales como } \\
\text { fisicos. }\end{array}$ \\
\hline $\begin{array}{l}\text { A20 } \\
\text { Asumir la } \\
\text { innovación } \\
\text { como un } \\
\text { continuo de la } \\
\text { mediación } \\
\text { pedagógica. }\end{array}$ & $\begin{array}{l}\text { Clarida } \\
\text { d: } \\
1,90= \\
1,90^{*} \\
\text { Importa } \\
\text { ncia } \\
1,99> \\
1,98^{*} \\
\text { Pertine } \\
\text { ncia: } \\
1,99>1 \text {, } \\
98^{*}\end{array}$ & $\begin{array}{l}\text { Clarida } \\
\text { d: } \\
0,29<0, \\
30^{*} \\
\text { Importa } \\
\text { ncia: } \\
0,11<0 \text {, } \\
13^{*} \\
\text { Pertine } \\
\text { ncia: } \\
0,11<0 \text {, } \\
14^{*}\end{array}$ & $\begin{array}{l}\text { Claridad: } \\
1,95> \\
1,93 \\
\text { Importan } \\
\text { cia 1,98> } \\
1,97 \\
\text { Pertinen } \\
\text { cia: } \\
1,98>1,9 \\
7\end{array}$ & $\begin{array}{l}\text { Clarida } \\
\text { d: } \\
0,22<0 \text {, } \\
24^{*} \\
\text { Importa } \\
\text { ncia: } \\
0,13<0 \text {, } \\
15^{*} \\
\text { Pertine } \\
\text { ncia: } \\
0,13<0 \text {, } \\
16^{*}\end{array}$ & $\begin{array}{l}\text { La } \\
\text { función } \\
\text { es } \\
\text { clara, } \\
\text { muy } \\
\text { importa } \\
\text { nte y } \\
\text { muy } \\
\text { pertinen } \\
\text { te. }\end{array}$ & $F 2=755$ & $\begin{array}{l}\text { No se realizaron modificaciones } \\
\text { debido a que la función se } \\
\text { encontraba dentro de los } \\
\text { parámetros de rigurosidad } \\
\text { establecidos. }\end{array}$ \\
\hline
\end{tabular}

Elaboración propia, 2020

${ }^{*}$ Corresponde a la media general de todas las funciones

A pesar de que se había contemplado inicialmente eliminar funciones, el conjunto de resultados obtenidos hizo que el grupo de personas investigadoras se decantara principalmente por fusionar ítems en algunos casos, como se presentó con la función C4.

Es importante acotar en este apartado que una de las dificultades metodológicas experimentadas fue que se utilizó la misma codificación para las funciones contenidas en Q1, Q2 y Q3, por lo que, al hacer el contraste con el criterio de los representantes del MEP, fue necesario regresar al ítem para poder realizar la comparación, lo que hizo más lento y engorroso el proceso de análisis.

\subsection{Fase mixta e integradora}

Aunada a las fases anteriores, el grupo de investigadores contó con otros insumos para complementar el análisis -como se detalló en el apartado de este documento titulado Metodología-, tales como aportes dados en los cinco cuestionarios relacionados con aspectos a considerar en la redacción de las funciones. $\mathrm{O}$, en el caso particular del profesorado, este espacio se constituyó en una oportunidad para externar sus apreciaciones alrededor de la educación inclusiva, las normativas, sus temores y necesidades. Además, se contaba con los hallazgos consignados en el informe final de un seminario de graduación realizado por profesoras y estudiantes de las carreras de Preescolar y Educación Especial de la UNA, sobre las funciones que realiza 
in situ el profesorado de estas especialidades (Cordero et al., 2019), así como con el informe de la revisión realizada por Zúñiga (2020) sobre observaciones y recomendaciones dadas por pares evaluadores externos a las carreras nacionales de Educación Especial sometidas a procesos de acreditación por parte del SINAES.

Una vez constituidas las funciones de la fase cuantitativa, el grupo investigador las contrastó con los hallazgos de los insumos mencionados; así logró establecer que la mayoría de las características identificadas en estos documentos ya estaban incluidas en las funciones del nuevo perfil, lo que daba mayor fiabilidad a las funciones construidas como producto de todo el proceso de estudio y análisis.

También fueron incorporados aspectos como la creatividad dentro de funciones asignadas a las dimensiones actitudinal y procedimental, el ejercicio transdisciplinar de la profesión en la definición de redes de apoyo familiar, comunitario e interpersonal, así como nuevos espacios emergentes en la labor docente y el concepto de ambiente sustentable como eje transversal en algunas de las funciones.

Se notó, además, que algunos elementos emergían por sí mismos de forma transversal en el perfil profesional, tales como la necesidad de contar con un profesional capaz de enfrentar los cambios del contexto y la disciplina, y la de reflexionar de manera constante sobre la relación teoría-práctica en la docencia.

El conjunto de procedimientos anteriores permitió establecer finalmente un perfil profesional de la EE costarricense de 88 funciones distribuidas como se detalla en la Tabla 5 (Meléndez et al., 2020).

TABLA 5

Distribución de las funciones por dimensión en el perfil profesional final

\begin{tabular}{lcccc}
\hline $\begin{array}{l}\text { Áreas de } \\
\text { competencia }\end{array}$ & $\begin{array}{c}\text { Dimensión } \\
\text { Conceptual }\end{array}$ & $\begin{array}{l}\text { Dimensión } \\
\text { Actitudinal }\end{array}$ & $\begin{array}{l}\text { Dimensión } \\
\text { Procedimental }\end{array}$ & $\begin{array}{l}\text { Total } \\
\text { por área }\end{array}$ \\
\hline $\begin{array}{l}\text { 1. Enfoques teóricos, } \\
\text { metodológicos, } \\
\text { políticos y legales }\end{array}$ & 7 & 6 & 6 & 19 \\
$\begin{array}{l}\text { 2. Investigación y } \\
\text { evaluación de la } \\
\text { práctica }\end{array}$ & 5 & 3 & 4 & 12 \\
$\begin{array}{l}\text { 3. Mediación } \\
\text { pedagógica }\end{array}$ & 8 & 10 & 8 & 26 \\
$\begin{array}{l}\text { 4. Desarrollo Humano } \\
\begin{array}{l}\text { 5. Redes de apoyo } \\
\text { familiar, comunitario, } \\
\text { interprofesional }\end{array}\end{array} \quad 5$ & 5 & 3 & 13 \\
$\begin{array}{l}\text { 6. Gestión, } \\
\text { administración, } \\
\text { desarrollo de } \\
\text { proyectos }\end{array}$ & 7 & 2 & 4 & 13 \\
\hline \multicolumn{1}{c}{ TOTAL } & 1 & 1 & 3 & \\
\hline
\end{tabular}


La tabla anterior muestra que el área de competencia Asesoramiento: familiar, comunitario, institucional se integró al área Redes de apoyo familiar, comunitario, interpersonal, pasando así de tener 7 áreas de competencia a 6 en el perfil final. Además, la categoría Mediación pedagógica fue la que sufrió mayores cambios en las dimensiones conceptual y procedimental. A pesar de que la dimensión conceptual tiene una mayor cantidad de funciones, se puede evidenciar que el perfil incorpora las tres dimensiones en una forma equilibrada.

\section{CONCLUSIONES Y RECOMENDACIONES}

La política educativa actual, que tiende hacia la educación inclusiva, requiere profesionales con formación adecuada y efectiva para derribar las barreras del aprendizaje, la participación y el éxito de la población estudiantil que participa del sistema educativo costarricense. No obstante, en Costa Rica, a la fecha en que se publica el informe de esta investigación no existía un perfil profesional para la EE que se ajustara a los cambios paradigmáticos mundiales y nacionales en educación inclusiva; por lo tanto, cada centro formador, desde su experiencia y posición epistemológica, ha establecido lo que consideraba debía ser el perfil de salida sin un referente nacional en cuanto al ejercicio de la profesión. Por ello, el producto de este estudio se constituye en una herramienta válida y útil para los propósitos para los que fue desarrollado, que tienen que ver con el diseño de perfiles de formación para la organización de actividades de actualización profesional, para guiar la construcción de instrumentos para la selección y reclutamiento de personal docente en el área o para la evaluación del desempeño profesional, y para impulsar nuevas investigaciones, amén de otros usos que la sociedad pudiere identificar.

El ejercicio investigativo realizado se logra gracias al esfuerzo de diferentes sectores vinculados a la educación y formación de profesionales; por lo tanto, es necesario comprenderlo como un producto de construcción colectiva de actores con conocimiento y experiencia, tales como profesionales del área, usuarios de los servicios atendidos por esos profesionales, agencias acreditadoras de educación superior, colegios profesionales, familias y entidades vinculadas con la capacitación permanente y continua del profesorado en ejercicio. Además, por supuesto, es producto de la observación in situ de la labor profesional y de los expertos en educación de diversas asignaturas y niveles, en tanto se trata de un profesional de apoyo a los procesos educativos de todo el estudiantado.

Desde una perspectiva heurística, la experiencia recuperada permite afirmar que la unión en equipos interdisciplinarios y con aproximaciones metodológicas mixtas permite la construcción de perfiles profesionales con mayor rigurosidad y amplitud de criterio. También, analizar los productos parciales de cada fase y darles secuencialidad reorienta la toma de decisiones desde esa espiral metodológica que se expresa en la teoría investigadora para este tipo de estudios, en la que el complemento cuantitativo, además, ha llevado a evidenciar valores extremos que representan objetos de importancia para una validación, en este caso, para la mejor definición de las funciones que debían integrar el perfil.

De esta manera, el perfil inicial que contaba con 98 funciones y 7 áreas de competencia pasó a tener un producto final de 88 funciones distribuidas en 6 áreas de competencia, que además transitó por una reestructuración de funciones y mejoramiento de la redacción para mayor claridad, inclusión de elementos que permitieron mejorar la pertinencia contextual ajustada a las nuevas tendencias disciplinares y un reacomodo de funciones en cuanto a la dimensión o área de competencia que mejor las integraba. Estos aspectos se constituyeron así en las características explicativas del proceso de validación, gracias al criterio experto de los informantes validadores que colaboraron con esta investigación.

Así, y como ya se dijo, entre otros usos, el perfil resultante es una base para que las distintas instituciones formadoras orienten la toma de decisiones curriculares para la formación y actualización de los profesionales con miras a responder a las exigencias del medio laboral docente en concordancia con las acciones inclusivas requeridas. Sin embargo, en adelante, uno de los retos identificados será que la sociedad costarricense 
reconozca la necesidad de que exista voluntad política para que se asuman las funciones del perfil profesional en EE para la evaluación, selección y contratación docente.

Sería ideal que existieran perfiles profesionales en todas las áreas de la educación costarricense, debidamente vinculados a la educación inclusiva, como una tarea de todos quienes integran el sistema educativo. Por esto, se espera que ese perfil se constituya en un modelo a seguir para la constitución de los demás y que a su vez funcione como herramienta de consulta que permita articular las decisiones y prácticas docentes por la educación inclusiva, entre el profesorado de apoyo y los demás actores del sistema educativo.

Como recomendaciones, es necesario acotar que el perfil profesional no es un producto acabado, en tanto no lo es la educación, sino que deberá transformarse en atención a las demandas de los cambios culturales y de las nuevas tendencias, por lo que se recomienda estar atentos a lo que ocurre en los contextos internacional y nacional. Además de continuar investigando, en procura de novedades y análisis confirmatorios del contenido del perfil profesional de EE. También es necesario revisar previamente la definición de las escalas a utilizar según lo que realmente interesa al valorar la función.

Dentro de los retos que asume el ONEI, como próximas tareas a desarrollar está la de operacionalizar cada función en resultados de aprendizaje, que permitan, de alguna manera, elevar y unificar la calidad profesional de quienes están llamados a trabajar por la educación inclusiva mediante el derribamiento de barreras al aprendizaje, la participación y el éxito escolar de todo el estudiantado.

Finalmente, como limitaciones de la investigación realizada se tiene que la participación en todas las fases de construcción del perfil fue voluntaria e intencional, y no incluyó el criterio de otros grupos de profesionales que tuvieran relación directa o indirecta con el profesorado de EE.

\section{REFERENCIAS}

Alfaro, R. G., y Alvarado, C. S. (2018). El Perfil de profesores universitarios de universidades públicas y privadas en la carrera de Educación. Actualidades Investigativas en Educación, 18(2), 263-284. DOI: https:// doi.org/10.15517/aie.v18i2.33161

Almenta, E. y Leiva, J. (2018).Formación inicial del profesorado para una escuela inclusiva: el aprendizaje profesional de los estudiantes del grado de educación primaria. Ponencia presentada en el Congreso internacional XXIX Jornadas de Universidades y EE Prácticas en Educación Inclusiva: Diálogos entre Escuela, Ciudadania y Universidad (pp. 908-916). DOI: 10.13140/RG.2.2.11447.83369

Benavides, O., y Gómez-Restrepo, C. (2005). Métodos en investigación cualitativa: triangulación. Revista Colombiana de Psiquiatría, vol. XXXIV, 1, 118-124.

Bernal, P. y Arango, M. (2017). CUESTIONARIOS. En Bernal P. (Ed.), La Investigación en Ciencias Sociales: Tecnicas de recolección de la información (pp. 55-80). Universidad Piloto. doi:10.2307/j.ctv7fmfjk.8

Castillo, K., Deliyore, R., González, V., Madriz, L., Marín, M.G., Meléndez, L., Montenegro, F., Rodríguez, R. Segura, M., y Solórzano, J. (mayo-agosto 2018). Análisis epistemológico de la Educación Especial en Costa Rica. Actualidades Investigativas en Educación, 8(2),1-28. DOI: https://doi.org/10.15517/aie.v18i2.33066

Cordero, M., López, E., Espinoza, J., Ferreto, S., Herrera, A., León, M. J., Marín, I., Mata, Y., Méndez, K., Monge, P., Mora, V., Torres, Y., Chaves, A. L., y Dobles, C. (2019). Mirada del quehacer de las profesionales graduadas de las carreras de Pedagogía con énfasis en Educación Preescolar y EE, al asumir el reto de abordar la diversidad de su quehacer pedagógico desde la experiencia laboral. Informe de Seminario de Graduación. Heredia: CIDE/UNA.

Creswell, J. (2003). Outline: Creswell's Research Design (3a ed.). [Esquema: Diseño de investigación de Creswell]. United States of América: SAGE publications.

Godoy, M., Otondo, M. y Rey, V. (2016). Cap. 3 Formación de profesores/profesoras diferenciales: Desde la mirada de las Universidades pertenecientes al Consejo de Rectores de Chile. En V. Alves y R. Vargas (Coords.), Educación inclusiva en América Latina: politicas, investigaciones y experiencias, 66-89. Brasil: Intertexto y México: CAPUB. 
Gurdían, A. (2007). El paradigma cualitativo en la investigación socio educativa. Coordinación Educativa y Cultural Centroamericana. Agencia Española de Cooperación Internacional. San José: Printcenter.

Hernández-Sampieri, R., Fernández Collado, C. y Baptista Lucio, P. (2014). Metodología de la Investigación. (Sexta Edición). México: McGraw-Hill/Interamericana Editores, S.A. d C.V. ISBN:978-1-4562-2396-0

López-Aguado, M., y Gutiérrez-Provecho, L. (2019). Cómo realizar e interpretar un análisis factorial exploratorio utilizando SPSS. REIRE Revista d'Innovació i Recerca en Educació, 12(2), 1-14. http://doi.org/10.1344/ reire2019.12.227057

Martínez-Izaguirre, M., Villardón, G. L., y Yániz, A. C. (2013). Elaboración y validación del perfil de competencias docentes en educación obligatoria. En International Conference Re-conceptualizing the professional identity of the European teacher. Sharing Experiences (pp. 551-562). Sevilla: Copiarte. Recuperado de http:// hdl.handle.net/11441/56725

Meléndez, L. (2005). La Educación Especial en Costa Rica: fundamentos y evolución. Costa Rica: EUNED.

Meléndez, L., Abarca, V., Deliyore, R., Hernández, E., Quesada, J., Ramírez, R., Rodríguez, M., Rojas, M., Segura, M., Sibaja, Z., Solís, G., Solórzano, J., Vargas, M., y Zúñiga, S. (2020). Informe: Diseño y validación del perfil profesional de la EE costarricense. San José: COLYPRO. Recuperado de https://www.researchgate.net/ publication/347271165_DISENO_Y_VALIDACION_DEL_PERFIL_PROFESIONAL_DE_LA_EDUCACION_ESP

Meléndez, L. (2020). Tendencias formativas en Educación Especial en Iberoamérica. Revista Colombiana de Educación, $79,267-288$.

Ministerio de Educación Pública. (2016). Política Curricular 2016. Bajo la visión de educar para una nueva ciudadanía. San José: MEP. Recuperado de https://www.mep.go.cr/politica-curricular.

Morales, P. (2011). El análisis factorial en la construcción e interpretación de test, escalas y cuestionarios. Universidad Pontificia Comillas, Madrid, pp. 1-45. Recuperado de http://www.upcomillas.es/personal/peter/investigacion/ AnalisisFactorial.pdf

OCDE. (2017). Educación en Costa Rica, Reseñas de Políticas Nacionales para la Educación. París: Oecd Publishing. Recuperado de https://doi.org/10.1787/9789264277335-en.

Ortiz, H. (2007). Formación del profesorado y atención a la diversidad: desafíos a los procesos de innovación curricular en educación inicial. UCMaule-Revista Académica de la Universidad Católica del Maule, 33, 45-60. Recuperado de http://search.ebscohost.com/login.aspx? direct $=$ true $\& \mathrm{db}=\mathrm{a} 9 \mathrm{~h} \& \mathrm{AN}=36216615 \&$ lang $=\mathrm{es} \&$ site $=$ eds-live \&scope $=$ site

Programa Estado de la Nación, PEN. (2017). Sexto informe estado de la educación / PEN. -- 1 ed. -- San José C. R: Servicios Gráficos, A. C. 432 páginas. ISBN-978-9930-540-02-2 -impreso ISBN-978-9930-540-04-6 -digital. http://hdl.handle.net/20.500.12337/665

Tineo, L.E. (2012).Validación del perfil profesional basado en competencias de docentes de institutos de educación superior tecnológico y su grado de aceptación de profesores, a nivel nacional (Tesis de maestría), Universidad Nacional Mayor de San Marcos, Perú. Recuperado de http://cybertesis.unmsm.edu.pe/handle/cybertesis/1682

UNESCO. (2015). Incheon Declaration. Education 2030: Towards inclusive and equitable quality education and lifelong learning for all [Hacia una educación de calidad inclusiva y equitativa y un aprendizaje permanente para todos]. Recuperado de http://www.unesco.org/new/fileadmin/MULTIMEDIA/HQ/ED/ ED/pdf/FFA_Complet_Web-ENG.pdf

Villalobos, L. (2017). Enfoques y diseños de investigación social: cuantitativos, cualitativos y mixtos. San José: EUNED.

Zúñiga, S. (2020). Sistematización de las fortalezas y recomendaciones encontradas en la evaluación externa y evaluación de informes de logro del Proyecto Especial de Mejoras de parte del SINAES, en cinco carreras de EE evaluadas en Costa Rica del año 2011 al 2019. pp. 1-11, (inédito). San José, Costa Rica. 